Und was, meine Damen und Herren, können nun Frauen selbst tun, um ihre Talente und Stärken in Wirtschaft und Gesellschaft besser zur Geltung zu bringen?

Ich denke, Frauen müssen lernen, Chancen nicht nur zu sehen, sondern auch für sich zu nutzen bzw. zu beanspruchen.

Dazu gehört, dass sie ihre wertvollen Kompetenzen hervorheben und ins Unternehmen einbringen - und dass sie aktiv an ihrer Persönlichkeit arbeiten.

\section{Stichwort: Statussymbole}

Manchmal werde ich gefragt: Wie sieht denn der richtige Weg in der Führung aus? Ich sage: diesen einen richtigen Weg gibt es nicht! Jede Frau sollte sich auf ihren eigenen Weg konzentrieren.

Meine Empfehlung an alle Damen:

- Blicken Sie öfter mal über den eigenen „Tellerrand“ Ihres Arbeitsplatzes hinaus!
- Suchen Sie das Gespräch mit anderen und tauschen sich über Ihre Gedanken aus!

- Knüpfen Sie Netzwerke - schließen Sie Freundschaften seien Sie offen für Dialoge - suchen Sie sich Ratgeber und hinterfragen Sie sich selbst!

Jeder Mensch sollte sich auch immer wieder bewusst machen: Man trägt nicht nur Verantwortung für andere - man muss auch Verantwortung für sich selbst übernehmen.

Aus meiner 40-jährigen Berufserfahrung habe ich gelernt, was es bedeutet, Freiraum und Gestaltungsmöglichkeiten zu nutzen:

Ich habe auf der ganzen Welt viele unglaublich interessante Menschen kennengelernt und immer wieder erlebt, was Sinnerfüllung heißt.

Und meine wichtigste Erfahrung war immer wieder: Letztendlich gewinnt die Menschlichkeit.

Vielen Dank.

\title{
Vorstellung der Studie „Aktionärinnen fordern Gleichberechtigung“
}

\section{Dezember 2010, Bertelsmann Repräsentanz, Berlin}

\section{Prof. Dr. Marlene Schmidt}

Vorsitzende der Kommission Arbeits-, Gleichstellungs- und Wirtschaftsrecht des djb; Rechtsanwältin, Frankfurt a.M.

Meine sehr verehrten Damen und Herren,

in dieser 129-seitigen Veröffentlichung finden sich neben der Auswertung unseres Projekts „Aktionärinnen fordern Gleichberechtigung “ eine Fülle von Überlegungen, wie eine wirksame Erhöhung des Frauenanteils in Führungspositionen - insbesondere Aufsichtsratspositionen - deutscher Unternehmen bewerkstelligt werden kann. Ich möchte Ihnen im Folgenden zunächst die Auswertung der Hauptversammlungsteilnahmen vorstellen. Anschließend greife ich beispielhaft einige der sonstigen Beiträge heraus, um deutlich zu machen, welche kreativen Ideen die Mitglieder des Deutschen Juristinnenbunds entwickelt haben in der Hoffnung, dass möglichst viele von ihnen aufgegriffen und umgesetzt werden.

\section{Das Projekt „Aktionärinnen fordern Gleichberechtigung“}

Ziel des Projekts war es nicht in erster Linie, empirische Daten zu erheben, sondern das Thema Frauen in Führungspositionen sollte durch die Fragen in den Hauptversammlungen über die Anteilseigner und die Presse eine größere Öffentlichkeit erreichen und es sollte eine Sensibilisierung für dieses Thema stattfinden. Bei den Hauptversammlungen wurden Fragen zu Diversity und konkreten Maßnahmen zur Förderung von Frauen in Führungspositionen gestellt. Weiterhin wurde nach Frauenförderprogrammen und Veröffentlichungen von frau- enspezifischen Daten und Kennziffern im Lagebericht gefragt sowie die Ansichten der Entscheidungsträger, d.h. von Vorstand und Aufsichtsrat, zur Erhöhung des Frauenanteils in ihrem Unternehmen eingeholt.

Durch wiederholte Teilnahme an den Versammlungen und Stellen dieser Fragen sollte ein Rechtfertigungsdruck aufgebaut werden. So wird es möglich sein, Aussagen von Vorstand und Aufsichtsrat derselben Unternehmen an ihren Angaben vom Vorjahr zu messen. Von Januar bis Juli 2010 besuchten 72 Mitglieder des djb, der ARGE Anwältinnen sowie Interessentinnen anderer Professionen, darunter auch eine Journalistin, eine Ingenieurin und eine Psychologin, die Hauptversammlungen aller DAX30- und weiterer 44 Unternehmen aus dem TecDAX und MDAX. Alle Besuche erfolgten ehrenamtlich.

Ausgangspunkt des Projektes „Aktionärinnen fordern Gleichberechtigung“ ist die uneingeschränkte Entsprechenserklärung in Bezug auf Diversity, die von sämtlichen DAX30sowie den weiteren vom djb besuchten 44 TecDAX- und MDAX-Unternehmen abgegeben wurde. Alle vom djb besuchten Unternehmen sind börsennotiert und damit verpflichtet, eine Entsprechenserklärung nach $\mathbb{S} 161 \mathrm{AktG}$ abzugeben. Sie müssen erklären, inwieweit sie die Empfehlungen des Deutschen Corporate Governance Kodex (DCGK) vom 18. Juni 2009 zur Besetzung von Aufsichtsrat und Vorstand eingehalten haben oder von ihnen abweichen. Dabei ist unter Diversity nach Angaben der Regierungskommission auch die Berücksichtigung von Frauen zu verstehen. 
Vorstand und Aufsichtsrat aller DAX30-Unternehmen sowie aller weiterer 44 besuchten börsennotierten Aktiengesellschaften aus dem TecDAX und MDAX haben die Entsprechenserklärung uneingeschränkt abgegeben. Die Unternehmen haben damit erklärt, bei der Besetzung von Vorstand und Aufsichtsrat auf Diversity geachtet zu haben.

Angesichts der geringen Präsenz von Frauen in Aufsichtsräten, Vorständen sowie in Führungspositionen generell und ausgehend von dieser Entsprechenserklärung sind alle DAX30-Unternehmen sowie weitere 44 börsennotierte Aktiengesellschaften von den Teilnehmerinnen auf den Hauptversammlungen dazu befragt worden, was genau sie unter Diversity verstehen, welche konkreten Maßnahmen sie ergreifen wollen, damit bei der nächsten Wahl auch Frauen berücksichtigt werden, wie hoch der Belegschaftsanteil von Frauen auf der ersten, zweiten, dritten und vierten Ebene unterhalb des Vorstandes ist, ob es Kennziffern gibt etc.

\section{Das Ergebnis}

Nur acht (26,66 Prozent) der befragten DAX-30Unternehmen können konkrete Maßnahmen zur Beachtung von Frauen bei der nächsten Bestellung von Vorstandsmitgliedern und Aufsichtsräten benennen. Allerdings stehen 43 Prozent der Unternehmen konkreten Maßnahmen positiv gegenüber, nur 6,66 Prozent negativ, alle übrigen sind neutral. Auffällig ist jedoch, dass vor allem konkrete Pläne nicht genannt wurden, sondern eine passive Haltung erkennbar ist. Gesprochen wurde von „sich Mühe geben“, „sich Gedanken machen“, sogar von „Herzensangelegenheit“. Eine solche passive Haltung ist nicht zielführend. Diese Haltung verdeutlicht, dass die meisten DAX30-Unternehmen weder Strategien noch konkrete Pläne haben, Diversity herzustellen.

Nur 6,81 Prozent der 44 befragten TecDAX und MDAX-Unternehmen konnten konkrete Maßnahmen zur Beachtung von Frauen bei der nächsten Bestellung von Vorstandsmitgliedern und Aufsichtsräten benennen. Ähnlich wie bei den DAX30Unternehmen äußerten sich 43,18 Prozent positiv zu solchen Maßnahmen, 9,09 Prozent negativ und 29,54 Prozent neutral. Auch hier fiel bei den positiven Äußerungen die Passivität auf: Explizit hieß es, sie würden auf Vorschläge warten, Frauen hätten immer eine Chance, sie seien offen für Frauen.

Die - neutralen - Angaben, es gäbe keine konkreten Planungen, eine Auseinandersetzung damit habe noch nicht stattgefunden oder es gäbe derzeit keine Notwendigkeit, keinen aktuellen Handlungsbedarf (achtmal genannt) zeigen, dass die Mehrzahl der Unternehmen keine Maßnahmen zur Er- höhung des Frauenanteils in ihre Planungen aufgenommen haben. Die Unternehmen haben sich an die „Vereinbarung zwischen der Bundesregierung und den Spitzenverbänden der deutschen Wirtschaft zur Förderung der Chancengleichheit von Frauen und Männern in der Privatwirtschaft" aus dem Jahr 2001 nicht gehalten. Es fehlen nicht nur Ergebnisse, sondern bereits konkrete Ansätze, Ziele und Maßnahmen, um diese Ziele zu erreichen. Die Haltung der Unternehmen ist vorwiegend passiv. Transparenz und Fortschritt sind nicht zu erkennen. Es gibt weder Sanktionen für die Nichtbeachtung von Frauen, noch ist der öffentliche Druck stark genug, um überhaupt ein Umdenken und Handeln zu erreichen.

\section{Das Thema Aufsichtsrätinnen müsste bis 2018 weitest- gehend machbar sein, dann liegen die beiden großen Wahltermine 2013 und 2018 hinter uns.}

Prof. Dr. Klaus-Peter Müller

Die DAX30-Unternehmen gaben zu 47 Prozent an, den Vorstand mehr intern oder zumindest zur Hälfte mit internen Bewerbern zu besetzen, bei den übrigen Unternehmen zeichnet sich ebenfalls dieser Trend ab, bei einer Quote von 30 Prozent. Hieraus lässt sich der Schluss ziehen, dass Unternehmen den Frauenanteil in den Führungsebenen unter dem Vorstand erhöhen müssen, um Frauen für den Vorstand bestellen zu können.

Der Frauenanteil in der Belegschaft der DAX30Unternehmen schwankt stark zwischen elf und 61

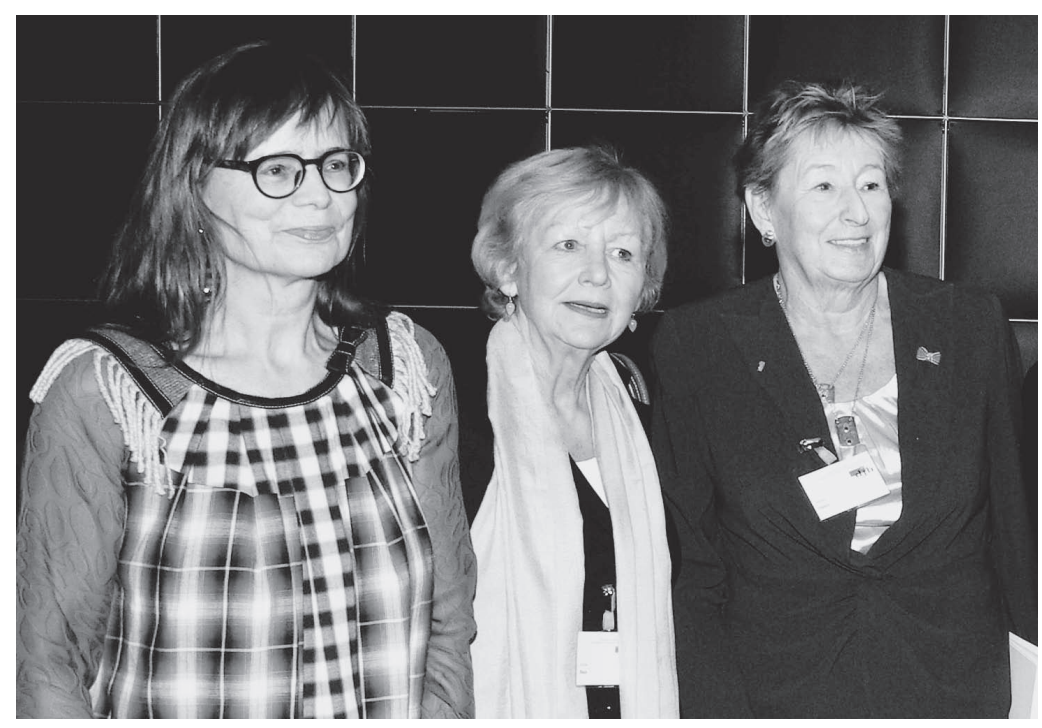

$\Delta$ Rechtsanwältinnen Mechtild Düsing (in Vertretung für DAV-Präsident Wolfgang Ewer), Ursula Raue (1. Vorsitzende des djb von 1993 bis 1997) und Renate Damm (1. Vorsitzende des djb von 1983 bis 1989, Ehrenpräsidentin) (v.l.n.r.).
Foto: @ Sharon Adler, Aviva-Berlin 
Prozent im Jahr 2009. Für die übrigen Unternehmen ergibt sich ein Intervall von acht bis 90 Prozent im selben Jahr. Zu den Antworten nach dem Frauenanteil in den Führungsebenen eins bis vier unterhalb des Vorstands für 2008 und 2009 fällt auf, dass nur drei der DAX30-Unternehmen Angaben zu allen Führungsebenen machten.

\section{Wir stehen vor einem riesigen Change-Prozess, was das Rollenbild von Frau und Mann angeht in unserer Gesellschaft, und die Änderung muss in den Köpfen} stattfinden. Bei jedem Change-Prozess ist wichtig, dass die Menschen im Boot sind. Ich habe aktuell noch nicht den Eindruck, dass alle in einem Boot sind.

Sabine Kauper

Von den übrigen 44 Unternehmen beantworteten die Frage sogar nur zwei Unternehmen vollständig. Nur fünf der 74 Unternehmen konnten somit für 2008 und 2009 den Frauenanteil für alle Führungsebenen benennen. Da diese Fragen fast allen Unternehmen ca. eine Woche vorher schriftlich angekündigt wurden und dennoch sehr unvollständige oder gar keine Angaben gemacht wurden, kann davon ausgegangen werden, dass die Daten entweder nicht vorhanden sind oder aber die Zeit nicht ausgereicht hat, um diese Zahlen vollständig zu generieren oder - wenn die Zahlen vorhanden waren sie nicht transparent gemacht werden sollten. So bat ein Unternehmen in seinem Antwortschreiben im Herbst 2009 ausdrücklich darum, die Daten nur in anonymisierter Form zu verwenden. Sofern die Unternehmen Zahlen zusammengefasst haben, ist

\section{Aus Sicht des Justizministeriums ist der system- \\ immanente Weg, über Fragerecht Druck auszuüben und Transparenz zu schaffen, richtig.}

\section{Dr. Birgit Grundmann}

nicht erkennbar, ob dies organisatorische wie zum Beispiel Umstrukturierung oder andere Gründe hat. Nur vereinzelt wurde angegeben, das Unternehmen habe eine flache Hierarchie, die vierte Ebene gebe es deshalb nicht. Die sich aus den Befragungen ergebende Datenmenge, die für einen Vergleich zur Verfügung steht, ist zu gering, um Schlüsse zu ziehen, ob - und wenn ja, wie - sich der Anteil an Frauen in Führungspositionen verändert hat. Bei den fünf Unternehmen mit vollständigen Daten ist innerhalb der einzelnen Ebenen sowohl ein Anstieg als auch ein Abfall der Anzahl von Frauen in Führungspositionen von 2008 auf 2009 zu verzeichnen. Daher lassen sich keine Aussagen über einen einheitlichen Trend machen. Erkennbar ist jedoch, dass sich der Anteil von Frauen an der Belegschaft bei den DAX30-Unternehmen auch nicht annähernd auf den Führungsebenen abbildet - wenige Unternehmen ausgenommen. Dies gilt auch für die 44 TecDAX- und MDAX-Unternehmen. Der Umgang mit frauenspezifischen Zahlen zeigt, dass es keinen einheitlichen Standard gibt, diese Zahlen zu erheben, und damit auch keine Vergleichbarkeit hergestellt werden kann. Festzustellen ist jedoch, dass generell der Anteil von Frauen in der Belegschaft nicht auf die oberen Führungsebenen transportiert wird und sich selbst auf den unteren Führungsebenen nur selten abbildet.

73 Prozent der DAX30- und 79 Prozent der 44 TecDAX- und MDAX-Unternehmen gaben an, keine Kennziffern dafür zu haben, wie der Frauenanteil auf den Führungsebenen in den nächsten fünf Jahren erhöht werden soll. Diese Tatsache allein zeigt, dass die Unternehmen für die Erhöhung des Anteils an Frauen in Führungspositionen keine gezielten Vorgaben haben. Um den Anteil von Frauen in Führungspositionen zu erhöhen, ist die Standortbestimmung jedes einzelnen Unternehmens erforderlich, es sind Kennziffern einzuführen, Ziele zu setzen und Maßnahmen zu ergreifen, damit diese Ziele erreicht werden. Diese Kennziffern sind zu beobachten, um kontrollieren zu können, ob die ausgewählten Maßnahmen geeignet sind, die gesetzten Ziele zu erreichen. Ohne Kennziffern und Ziele kann weder innerhalb des Unternehmens noch von außen messbar beurteilt werden, ob die Unternehmen sich für die Erhöhung des Anteils von Frauen in Führungspositionen aktiv einsetzen und ob dies zum Erfolg führt.

Jeweils annähernd die Hälfte der DAX30- und 44 TecDAX- und MDAX-Unternehmen äußerten sich ungefragt zur Frage der Sinnhaftigkeit einer Quote - alle negativ. Das am häufigsten geäußerte Argument war, die Qualifikation sei ausschlaggebend.

\section{Unsere Schlussfolgerungen}

- Die Unternehmen bekennen sich zu Diversity. Im Sinne einer angemessenen Beteiligung von Frauen wird Diversity in den Unternehmen allerdings nicht praktiziert. Unter Diversity wird ganz überwiegend auch die Beachtung von Frauen verstanden. Diese Beachtung wird mit der Entsprechenserklärung nach $\$ 161 \mathrm{AktG}$ bekundet, aber tatsächlich nicht oder kaum messbar umgesetzt. Nur wenige Unternehmen haben 
konkrete Maßnahmen ergriffen, um den Frauenanteil im Vorstand und im Aufsichtsrat zu erhöhen. Ebenfalls nur wenige Unternehmen haben Kennziffern und konkrete Zielvorgaben für die Erhöhung des Frauenanteils auf den vier Führungsebenen. Die Quote wird - soweit sich die Unternehmen dazu ungefragt geäußert haben - abgelehnt. Der Vorstand wird vorwiegend intern besetzt.

- Frauenspezifische Daten werden in den Unternehmen äußerst selten erhoben. Mangelnde Transparenz und fehlende Veröffentlichung führen dazu, dass ein Vergleich zwischen den Unternehmen nicht möglich ist und eine Entwicklung in den Unternehmen nicht überprüft werden kann.

- Der Anteil von Frauen in der Belegschaft wird gar nicht auf die obersten Führungsebenen transportiert und bildet sich auch in den vier Ebenen unter dem Vorstand kaum ab, obwohl 75 Prozent der Unternehmen angaben, flankierende Maßnahmen für die Vereinbarkeit von Familie/Privatleben und Beruf zu haben, die Mehrzahl der DAX30-Unternehmen auch spezielle Frauenförderprogramme zur Verfügung stellen und sich zumindest bei jüngeren Männern in Führungspositionen ein Mentalitätswechsel feststellen lässt, erkennbar daran, dass in 40 Prozent der Unternehmen Männer in Führungspositionen Elternzeit nehmen.

In Anbetracht dieser Befunde wird es Sie wenig verwundern, dass unser Fazit lautet:

Neun Jahre nach dem Abschluss der „Vereinbarung zwischen der Bundesregierung und den Spitzenverbänden der deutschen Wirtschaft zur Förderung der Chancengleichheit von Frauen und Männern in der Privatwirtschaft" fehlt es nicht nur an Ergebnissen, sondern bereits an Ansätzen wie Zielvorgaben, Kennziffern und konkreten Maßnahmen, um den Frauenanteil in Führungspositionen zu erhöhen. Flankierende Maßnahmen und spezielle Frauenförderprogramme sowie ein Mentalitätswechsel bei den jüngeren Männern haben nichts daran geändert, dass Frauen auf ihrem Weg in Führungspositionen nach wie vor an die "gläserne Decke“ stoßen, die trotz gegenteiliger Bekundungen seitens der Unternehmen immer noch ein fester Bestandteil des Unternehmensaufbaus ist. Sanktionen für die Nichtbeachtung von Frauen gibt es nicht. Einen Fortschritt wird es nur geben, wenn die Unternehmen ihre passive Haltung aufgeben und selbst aktiv werden. Dazu sollten sie sich Kennziffern geben, Ziele setzen sowie konkrete Maßnahmen benennen, wie diese Ziele erreicht werden sollen, Kontrollen der Ergebnisse mit Kon-

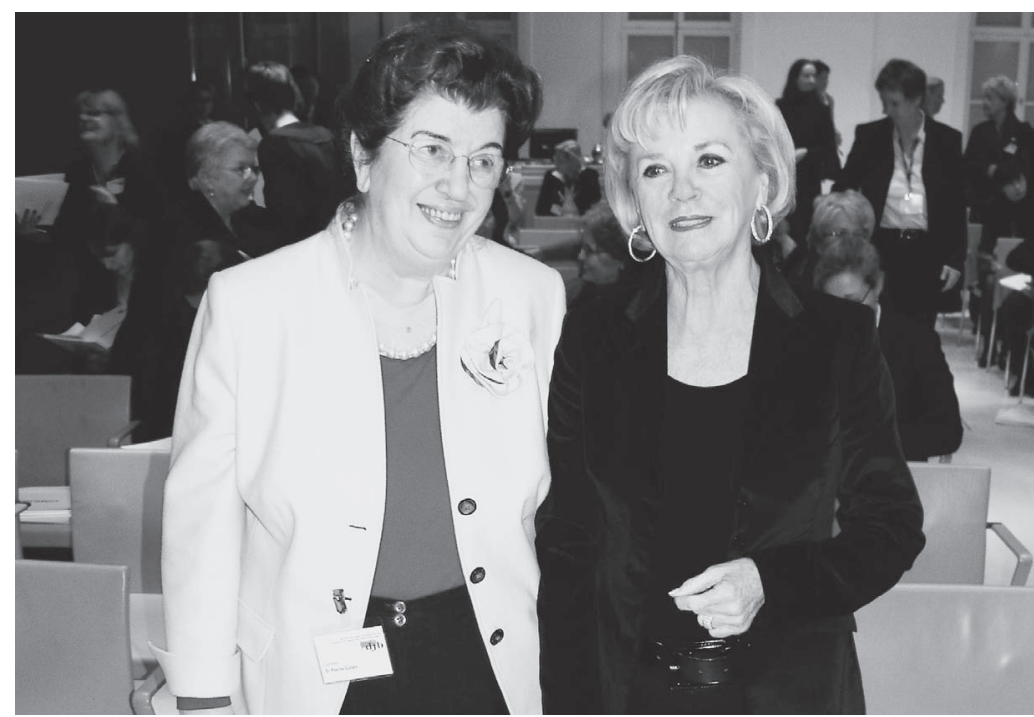

A Rechtsanwältin und Senatorin a.D. Dr. Lore Maria Peschel-Gutzeit (links) (1. Vorsitzende des djb 1977-1981, Ehrenpräsidentin) und Liz Mohn. $\quad$ Foto: @ Sharon Adler, Aviva-Berlin

sequenzen einführen - und dies sowie frauenspezifische Daten nachhaltig transparent machen.

\section{Rechtliche und betriebswirtschaftliche Rahmen- regelungen}

Neben den Ergebnissen der Aktion enthält die Studie Beiträge zum rechtlichen und betriebswirtschaftlichen Rahmen. Ein Beitrag befasst sich mit den Änderungen im Kodex. Am 26. Mai 2010 hat die Regierungskommission Deutscher Corporate Governance Kodex die Diversity-Empfehlung ergänzt, um den Anteil von Frauen und internationalen Vertreter(inne)n in deutschen Aufsichtsräten nachhaltig zu erhöhen. Zum Entwurf der Kodexänderung sagte Klaus-Peter Müller, Vorsitzender der Regierungskommission: „Frauenförderung ist

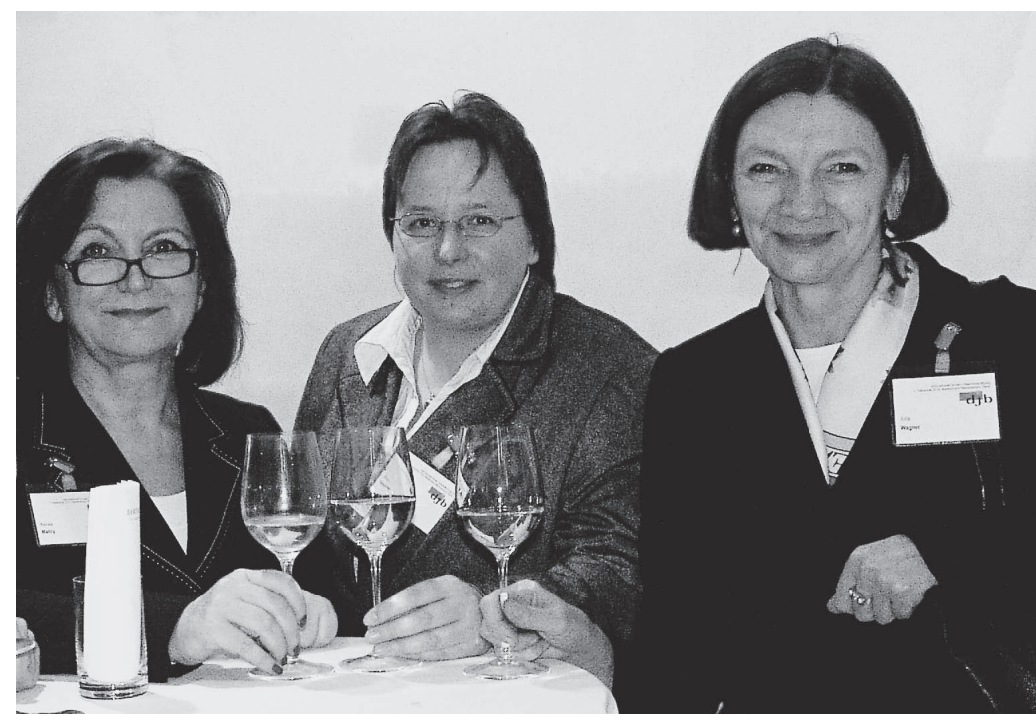

$\Delta$ Rechtsanwältinnen Renate Maltry (Vizepräsidentin des djb), Silvia Groppler (Vorsitzende der ARGE Anwältinnen im DAV), Jutta Wagner (Präsidentin des djb) (v.l.n.r.).

Foto: @ Sharon Adler, Aviva-Berlin 


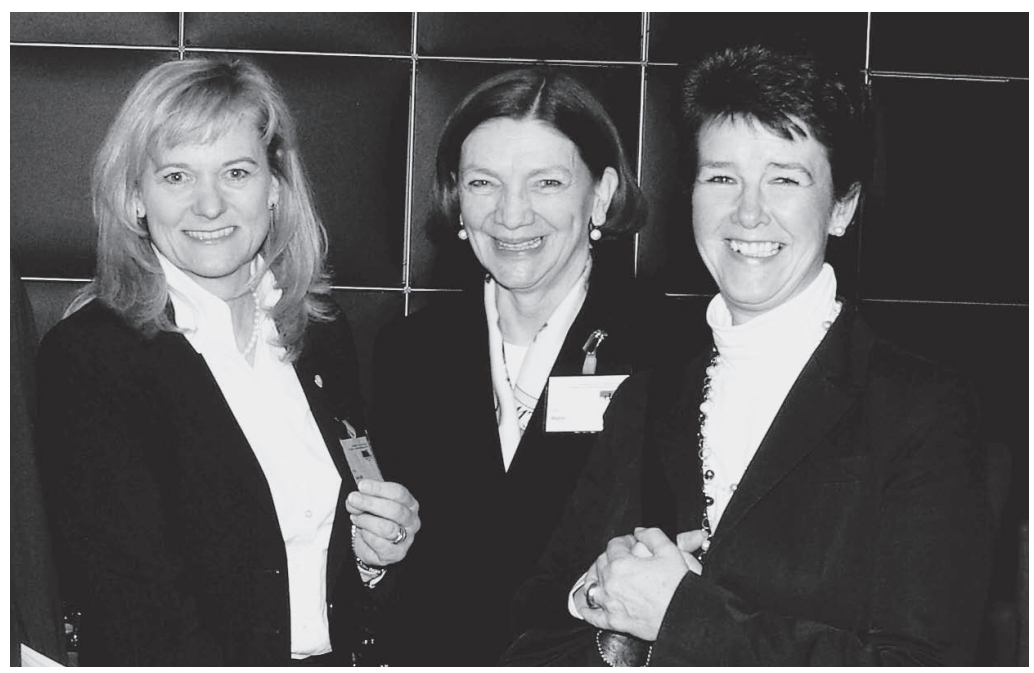

- Sylvia Canel MdB, Jutta Wagner (Präsidentin des djb), Dr. Birgit Grundmann (Staatssekretärin im BMJ) (v.l.n.r.).

Foto: @ Sharon Adler, Aviva-Berlin

nicht ein Tribut an den Zeitgeist, sondern liegt im ureigenen Interesse der Unternehmen. “ Der Einzug von Frauen in die Führungsebenen von Unternehmen ist nicht nur gesellschaftspolitisch wünschenswert, sondern auch unter ökonomischen Gesichtspunkten sinnvoll.

\section{Wir beobachten, dass sich bei dem Thema etwas tut. Es ist in der Wirtschaft viel breiter angekommen. Das ist auch ein Verdienst dieses Projektes. Der djb nutzt eine beste- hende Auskunftspflicht, um Transparenz zu schaffen.}

Thomas Fischer

Erweitert und ergänzt wurden die Empfehlungen in drei Punkten:

- Erweiterung auf die Führungspositionen. Wurde bisher Diversity nur für die Zusammensetzung von Vorstand und Aufsichtsrat gefordert, so wird diese Empfehlung jetzt auch auf die Führungspositionen im Unternehmen erstreckt.

\section{Quote kann natürlich nur heißen, dass, solange Frauen unterrepräsentiert sind, gleich gut geeignete Frauen ge- nommen werden müssen.}

Vera Gäde-Butzlaff

- Eine angemessene Berücksichtigung von Frauen ist anzustreben. Bezüglich der Bestellung des Vorstands und der Wahlvorschläge des Aufsichtsrats wurde bei der Diversity-Empfehlung der Halbsatz eingefügt, dass dabei „insbesonde- re eine angemessene Berücksichtigung von Frauen" angestrebt werden soll. Dies gilt ebenso für die Führungspositionen.

- Für die Zusammensetzung des Aufsichtsrats sind konkrete Ziele zu benennen,wobei die Ziele insbesondere eine angemessene Beteiligung von Frauen vorsehen sollen.

Die Kolleginnen haben in einem Beitrag der Studie untersucht, wie die Begriffe in ,auf Diversity ist zu achten" auszulegen sind, was die neue Empfehlung im Hinblick auf die Zusammensetzung des Aufsichtsrats beinhaltet und wie die Nichteinhaltung der Empfehlung sanktioniert wird. Sie kommen zu folgendem Ergebnis:

Der Aufsichtsrat muss konkrete Prozentzahlen oder absolute Zahlen für den Anteil von Frauen im Aufsichtsrat nennen, dokumentieren sowie in der Entsprechenserklärung offenlegen und begründen, wenn der Wahlvorschlag von der Zielvorgabe abweicht. Aufgrund der Unbestimmtheit der Kodexempfehlungen ist derzeit nicht absehbar, welche Ziele und welche Quote als den Empfehlungen entsprechend angesehen wird und welche nicht. Eine angegebene Quote ist zumindest grundsätzlich auf ihre Angemessenheit hin überprüfbar. Das Ausweichen auf allgemeine Angaben im Sinne einer Absichtserklärung, den Frauenanteil in absehbarer Zeit erhöhen zu wollen, um die Überprüfbarkeit zu vermeiden, ist allerdings keine Alternative. Denn in diesem Fall würde der Empfehlung des Kodex nicht entsprochen. Die Unternehmen sind deshalb aufgefordert, Farbe zu bekennen.

Ich selbst lege in einem Beitrag, der in weiten Strecken auf einem Gutachten beruht, dessen Erstellung das BMFSFJ finanziell gefördert hat, dar, dass die in $\mathbb{} 289$ Absatz 3 HGB verankerte Berichtspflicht über nichtfinanzielle Leistungsindikatoren wie Informationen über Umwelt- und Arbeitnehmerbelange, soweit sie für das Verständnis des Geschäftsverlaufs oder der Lage von Bedeutung sind, für unser Anliegen fruchtbar gemacht werden kann.

In dem Beitrag wird ausführlich und unter Berücksichtigung der europarechtlichen und der verfassungsrechtlichen Verpflichtung des Gesetzgebers, die tatsächliche Gleichstellung von Frauen und Männern auf allen Ebenen der betrieblichen und unternehmerischen Hierarchien zu fördern, begründet, dass sich bereits de lege lata gut vertreten lässt, dass der Anteil der Frauen an den Beschäftigten auf den verschiedenen Hierarchiestufen und Führungsebenen zu den „Arbeitnehmerbelangen“ im Sinne von $\$ 289$ Absatz 3 HGB gehört, über den regelmäßig zu informieren ist, weil dies 
für das Verständnis des Geschäftsverlaufs oder der Lage von Bedeutung ist.

Im Schlusskapitel der von Wirtschaft und Bundesregierung gemeinsam erarbeiteten „3. Bilanz Chancengleichheit“ heißt es insoweit ausdrücklich: „Die Förderung der Chancengleichheit von Frauen und Männern in der Privatwirtschaft wird auch künftig bilanziert und aufgezeichnet. Dabei werden weiterhin gezielt Indikatoren wie z.B. Frauen in Führungspositionen, Erwerbstätigenquoten oder Beteiligungen an Aus- und Weiterbildung erfasst und dargestellt." Leider dürfte diese Aussage nicht wörtlich zu verstehen sein. Gemeint ist vermutlich nur die Bilanzierung in einer allgemeinen, nicht unternehmensspezifischen „Bilanz Chancengleichheit". Dies ist jedoch völlig unzureichend.

Tatsächlich macht bislang kaum ein Unternehmen von der Möglichkeit Gebrauch, Auskunft zu geben über den Anteil der Frauen an den Arbeitnehmer(inne)n auf den verschiedenen Hierarchieebenen eines Unternehmens. Zusammenfassend ist daher festzustellen, dass die bestehenden Berichtspflichten keineswegs zur Folge haben, dass im Anhang zum Jahresbericht oder im Lagebericht tatsächlich über den Anteil der Frauen an den Führungskräften auf den verschiedenen Hierarchieebenen im Unternehmen oder gar über deren durchschnittliche Gehälter berichtet würde. Offenbar sind diese Punkte kein Thema für die Unternehmen. Dies dürfte zum einen darauf zurückzuführen sein, dass der Wortlaut des $\$ 289$ Absatz 3 HGB insoweit unpräzise und auslegungsbedürftig ist. Zum anderen ist die Frauenförderung offenbar anders als beispielsweise die Nachhaltigkeit - kein Thema, mit dem die Unternehmen für sich werben möchten und daher freiwillige Angaben machen. Das sollte sich ändern.
Ein besonders spannender Beitrag in der Studie befasst sich mit der Frage der Verfassungsmäßigkeit einer gesetzlichen Quotenregelung für die Besetzung von Aufsichtsräten. Es wird Sie nicht erstaunen, dass der djb der Auffassung ist - wie dies auch gründlich geprüft und in der Studie dargelegt wurde -, dass die Einführung einer Quote verfassungsmäßig wäre.

\section{Führungspositionen sind noch nicht weiblich besetzt und damit kann die nächste Stufe - Vorstand und Aufsichtsrat - gar nicht besetzt werden.}

Sabine Kauper

Weitere Themen, die in dieser Studie abgehandelt werden, sind:

- der Stand der Diskussion über Quotenregelungen in der Europäischen Union und

- in Bewertung der Ergebnisse von Studien, die die Auswirkung der Beteiligung von Frauen in der Unternehmensführung auf den Unternehmenserfolg untersucht haben.

- Ferner haben wir Handlungsempfehlungen erarbeitet, zu denen neben einer Empfehlung zur Novellierung des $\mathbb{2} 289$ HGB und zur Berücksichtigung der Erhöhung des Frauenanteils in Führungspositionen im Rahmen von Zielvereinbarungen von Vorstandsmitgliedern auch Strategien und Konzepte zur Veränderung der Unternehmenskultur gehören.

Ich hoffe, dass es mir gelungen ist, Ihre Neugier zu wecken, so dass Sie die Beiträge der Studie oder Teile davon nachlesen, und wünsche Ihnen und uns, dass Sie vieles davon interessant, bedenkensund umsetzungswert finden.

\section{Frauen an die Spitze! Frauen an die Spitze? \\ Empfehlungen für Karriereplanungen, ein Vortrag am 22. November 2010 in der Bucerius Law School in Hamburg}

\section{Renate Damm \\ Rechtsanwältin, Hamburg}

Im Sommer des vergangenen Jahres saß ich mit einigen Kolleginnen auf „Bodo‘s Bootssteg“ bei einem Glas Wein zusammen, nachdem wir zuvor unser Sommerfest mit einer Alsterdampferkreuzfahrt auf der Außenalster begangen hatten. Wir waren nur eine kleine Gruppe. Jede von uns erzählte ein wenig über das, was sie beruflich so mache und ich erwähnte, dass ich viele Jahre als Justitiarin im Axel Springer Verlag gearbeitet und im Jahre 2000 zusammen mit meinem Kollegen Dr. Mann die Anwaltskanzlei Damm \& Mann gegründet habe.

Im Verlaufe der Unterhaltung erzählte dann eine junge Kollegin, die auch als Justitiarin in einem Hamburger Unternehmen tätig ist, sie sei im letzten Jahr zusammen mit einem gleichfalls jungen Kollegen bei einem Seminar gewesen und habe auch in diesem Jahr wieder an dieser Fortbildungsver- 